\title{
Clinicopathological significance of ROCK1 and PIK3CA expression in nasopharyngeal carcinoma
}

\author{
XUANYI WANG ${ }^{1,2^{*}}$, YUXIANG HUANG ${ }^{3 *}$, RENHUA GUO $^{4}$, YONGBIAO LIU $^{5}$, \\ YAYUN QIAN ${ }^{2}$, DAN LIU $^{6}$, XIAOJUN DAI ${ }^{2}$, ZHENG WEI $^{2}$, FENG JIN $^{2}$ and YANQING LIU ${ }^{2}$
}

\begin{abstract}
${ }^{1}$ Rheumatology Department, Clinical Medical College, Yangzhou University, Yangzhou, Jiangsu 225001;
${ }^{2}$ Institute of Traditional Chinese Medicine and Western Medicine, School of Medicine, Yangzhou University, Yangzhou, Jiangsu 225009; ${ }^{3}$ Oncology Department, Clinical Medical College, Yangzhou University, Yangzhou, Jiangsu 225001; Departments of ${ }^{4}$ Oncology and ${ }^{5}$ Radiation Oncology, First Affiliated Hospital of Nanjing Medical University, Nanjing, Jiangsu 210029; ${ }^{6}$ Department of Pathology, Clinical Medical College, Yangzhou University, Yangzhou, Jiangsu 225001, P.R. China
\end{abstract}

Received November 26, 2015; Accepted November 25, 2016

DOI: $10.3892 / \mathrm{etm} .2017 .4076$

\begin{abstract}
The aim of the present study was to determine the expression of rho-associated coiled-coil-containing protein kinase 1 (ROCK1) and phosphatidylinositol-4,5-bisphosphate 3-kinase catalytic subunit $\alpha$ (PIK3CA) in nasopharyngeal carcinoma (NPC), and to assess the association between the expression of these proteins and the clinicopathological features of NPC. ROCK1 and PIK3CA expressions were assessed in a tissue microarray of sections prepared from the tumors of 81 patients with NPC using immunohistochemistry. Western blot analysis was used to detect ROCK1 and PIK3CA expression in NP69 and 5-8F cells. $\chi^{2}$ analysis revealed that upregulation of ROCK1 was significantly associated with advanced $\mathrm{N}$ stage $(\mathrm{P}=0.032)$ cancer and increased PIK3CA expression was significantly associated with advanced $\mathrm{N}$ stage $(\mathrm{P}=0.027)$ and TNM stage $(\mathrm{P}=0.019)$ cancer. Furthermore, ROCK1 expression was significantly positively correlated with PIK3CA expression $(\mathrm{P}=0.01)$. Western blot analysis demonstrated that levels of ROCK1 $(\mathrm{P}<0.001)$ and PIK3CA $(\mathrm{P}=0.015)$ were significantly higher in $5-8 \mathrm{~F}$ cells compared with NP69 cells. The results of the present study indicate that high levels of ROCK1 and PIK3CA expression may be associated with advanced stages in NPC.
\end{abstract}

Correspondence to: Professor Yanqing Liu, Institute of Traditional Chinese Medicine and Western Medicine, School of Medicine, Yangzhou University, 88 University South Road, Yangzhou, Jiangsu 225009, P.R. China

E-mail: liuyq@yzu.edu.cn

*Contributed equally

Key words: immunohistochemistry, nasopharyngeal carcinoma, rho-associated coiled-coil-containing protein kinase 1, phosphatidylinositol-4,5-bisphosphate 3-kinase catalytic subunit $\alpha$, tissue microarray

\section{Introduction}

Nasopharyngeal carcinoma (NPC) is a type of malignant epithelial cell tumor. The incidence and mortality of NPC in China is amongst the highest in the world (1). NPC is typically poorly differentiated and exhibits a tendency to metastasize and recur elsewhere, which may lead to failures in treatment $(2,3)$. Additionally, invasion and metastasis lead to mortality in many patients with NPC (4). Histologically, the majority of cases of NPC (95\%) are undifferentiated or poorly differentiated, and these histological types are able to spread and metastasize easily (5). It is therefore important to study the mechanisms of invasion in NPC to elucidate the pathogenesis and potential therapeutic treatments for this disease. Previous studies have identified a number of molecular markers that are associated with cell proliferation, invasion, differentiation and metastasis in NPC $(6,7)$; however, the molecular pathogenesis underlying the development and progression of NPC remain to be elucidated.

Rho-associated coiled coil-containing protein kinase (ROCK) is a serine/threonine protein kinase and RhoA downstream effector protein. In mammals, two isoforms of ROCK exist: ROCK1 and ROCK2 (8). Overexpression of ROCK1 induces reorganization of the cytoskeleton, and promotes the formation of stress fibers and dot matrix adhesion, thus regulating cell movement and migration (9). ROCK1 also serves an important role in the regulation and maintenance of cell migration (8).

The phosphatidylinositol-4,5-bisphosphate 3-kinase catalytic subunit $\alpha$ (PIK3CA) gene encodes the p110 $\alpha$ catalytic subunit of phosphatidylinositol 3-kinase (PI3K), a member of the PI3K/AKT pathway that is important for the regulation of cellular functions including metabolism, proliferation, angiogenesis, protein synthesis and apoptosis (10). It has previously been reported that activating mutation of the PIK3CA oncogene negatively affects the prognosis of non-small-cell lung cancer (11), which suggests that PIK3CA may act as a potential prognostic biomarker.

However, to date, the association between ROCK1 and PIK3CA in NPC has not been well characterized. In the present 
study, the expression of these proteins in NPC tissue microarray (TMA) sections was studied via immunohistochemistry (IHC) and protein levels in cells were evaluated using western blot analysis. The association between ROCK1 and PIK3CA protein expressions and the clinicopathological features of patients were assessed to determine the clinicopathological significance of ROCK1 and PIK3CA in NPC.

\section{Materials and methods}

Patients and preparation of NPC TMA. Archived formalin-fixed, paraffin-embedded undifferentiated NPC tissue blocks were prepared from 81 patients with NPC who underwent biopsy surgery between January 2012 and November 2014 at the Radiation Oncology Department of Fudan University Cancer Hospital (Shanghai, China). Firstly, a pathologist marked the areas of tumor on the tissue block subsequent to reviewing a hematoxylin and eosin-stained section from each specimen. Single 2-mm diameter core tissues were taken from each paraffin-embedded tissue and placed into a recipient block. A total of 81 tissue sample cores were arrayed in each single block. A retrospective chart review of all patients was performed to collect data on clinical characteristics, including age, sex, pathological features and disease stage ( $\mathrm{T}$ and $\mathrm{N}$ ) according to the 2010 TNM classification of the American Joint Committee on Cancer Staging system (12). The histological type of all samples was undifferentiated. Approval was obtained from the Ethics Committee of Fudan University Cancer Hospital and all patients provided written prior informed consent.

IHC. For IHC analysis, TMA sections were deparaffinized in xylene, and endogenous peroxidase activity was blocked with methanol and $3 \% \mathrm{H}_{2} \mathrm{O}_{2}$ for $15 \mathrm{~min}$. For antigen retrieval, 4- $\mu \mathrm{m}$ thick TMA sections were boiled in a pressure cooker at $\sim 120^{\circ} \mathrm{C}$ in citrate buffer (pH 6.0) (Beyotime Institute of Biotechnology, Haimen, China) for $3 \mathrm{~min}$. Tissues were incubated for $1 \mathrm{~h}$ at room temperature with the following primary antibodies: Monoclonal rabbit anti-ROCK1 (cat. no. ab45171; 1:200; Abcam, Cambridge, MA, USA) or monoclonal rabbit anti-PIK3CA (cat. no. ab152155; 1:200; Abcam). The secondary antibody used was goat anti-rabbit horseradish peroxidase (HRP) (cat. no. K500711; ready-to-use; Dako; Aligent Technologies, Inc., Santa Clara, CA, USA). Immunostaining of tissues was evaluated independently by two trained pathologists who were unaware of the clinical background of the samples. For each case, five random fields at $400 \mathrm{x}$ magnification were captured by an Olympus IX73 microscope (Olympus Corporation, Tokyo, Japan) and digital camera (Olympus Corporation) using CellSens Dimension software (version 1.9; Olympus Corporation).

ROCK1 and PIK3CA immunoreactivity were analyzed using a semi-quantitative scoring system in which only cytoplasmic membrane staining was considered. Based on the percentage of positive cells, the level of ROCK1 and PIK3CA expression was classified as $(+++)$ if $\geq 76 \%$ of cells were stained, $(++)$ if $26-75 \%$ of cells were stained, $(+)$ if $\leq 25 \%$ of cells were stained, and (-) if cells completely lacked membranous staining. Thus, $(-)$ and $(+)$ cases were classified as exhibiting low protein expression, whereas $(++)$ and $(+++)$ cases were classified as exhibiting high protein expression (Table I).

Cell culture. The human nasopharyngeal epithelial cell line NP69 and the NPC cell line 5-8F were donated by the Sun Yat-sen University Cancer Center (Guangzhou, China). Following passage, cells were grown for $48 \mathrm{~h}$ in RPMI-1640 medium (Gibco; Thermo Fisher Scientific Inc., Waltham, MA, USA), supplemented with $10 \%$ fetal bovine serum (Gibco; Thermo Fisher Scientific Inc.) at $37^{\circ} \mathrm{C}$ in an atmosphere containing 5\% $\mathrm{CO}_{2}$. Following passage, the NP69 cell line was maintained in keratinocyte serum-free medium supplemented with human epidermal growth factor and bovine pituitary extract (cat. no. 17005-042; Gibco; Thermo Fisher Scientific Inc.) in a humidified atmosphere containing $5 \% \mathrm{CO}_{2}$ at $37^{\circ} \mathrm{C}$ for $72 \mathrm{~h}$.

Western blot analysis. Cells were washed twice with ice-cold PBS and resuspended in $1 \mathrm{ml}$ lysis buffer $(25 \mathrm{mM}$ Tris- $\mathrm{HCl}$ $\mathrm{pH} 7.6,150 \mathrm{mM} \mathrm{NaCl}, 1 \%$ NP-40, $1 \%$ sodium deoxycholate, $0.1 \%$ SDS) (Thermo Fisher Scientific Inc.). Cell debris was removed by centrifugation $\left(12,000 \times \mathrm{g} ; 4^{\circ} \mathrm{C} ; 10 \mathrm{~min}\right)$ and the protein concentration of the supernatant was subsequently determined using the Bradford method (13). Aliquots that contained $30 \mu \mathrm{g}$ total protein were separated by SDS-PAGE (6-10\% gradient gels) and proteins were transferred onto polyvinylidine diflouride membranes (EMD Millipore, Billerica, MA, USA). Membranes were blocked with $5 \%$ bovine serum albumin (Biosharp, Hefei, China) and incubated at $4^{\circ} \mathrm{C}$ for $18 \mathrm{~h}$ with the following monoclonal antibodies: Rabbit anti-ROCK1 (cat. no. ab45171; 1:2,000; Abcam), rabbit anti-PIK3CA (cat. no. ab152155; 1:2,000; Abcam) and rabbit anti- $\beta$-actin (cat. no. ab8227; 1:1,000; Abcam). Membranes were subsequently washed three times with $0.1 \%$ Tween-20 in TBS and incubated for $2 \mathrm{~h}$ at room temperature with HRP-conjugated secondary antibody (cat. no. sc2301; 1:2,000; Santa Cruz Biotechnology, Inc., Dallas, TX, USA). Immunoreactive protein bands were visualized using an electrochemiluminescence system (GE Healthcare Life Sciences, Chalfont, UK). To quantify the protein expression by Western blotting analysis, densitometric analyses were performed using ImageJ version 1.46 software (imagej.nih.gov/ij/). Experiments were performed at least three times.

Statistical analysis. The associations between clinicopathologic variables and ROCK1 and PIK3CA protein expression were examined using the $\chi^{2}$ test. Continuous data were compared using a Student's t-test if the distribution was normal, or a one-way analysis of variance if distribution was asymmetrical. All data were analyzed using SPSS version 16.0 software (SPSS, Inc., Chicago, IL, USA). P<0.05 was considered to indicate a statistically significant difference. All P-values were based on two-tailed tests.

\section{Results}

ROCK1 and PIK3CA expression in NPC. Expressions of ROCK1 and PIK3CA in clinical NPC tissues were evaluated using IHC. Representative images of the immunohistochemical staining patterns are displayed in Fig. 1. ROCK1 and PIK3CA 
Table I. Association between ROCK1 and PIK3CA immunoexpression and various clinicopathological parameters in nasopharyngeal carcinoma.

\begin{tabular}{|c|c|c|c|c|c|c|c|}
\hline \multirow[b]{2}{*}{ Parameter } & \multirow{2}{*}{$\begin{array}{c}\text { Number of } \\
\text { cases }(n=81)(\%)\end{array}$} & \multicolumn{3}{|c|}{ ROCK1 } & \multicolumn{3}{|c|}{ PIK3CA } \\
\hline & & Low (\%) & High (\%) & P-value & Low (\%) & High (\%) & P-value \\
\hline Sex, n (\%) & & & & 0.490 & & & 0.553 \\
\hline Male & $59(72.8)$ & $41(69.5)$ & $18(30.5)$ & & $23(40.0)$ & $36(60.0)$ & \\
\hline Female & $22(27.2)$ & $17(77.3)$ & $5(22.7)$ & & $7(31.8)$ & $15(68.2)$ & \\
\hline Age, n (\%) & & & & 0.638 & & & 0.392 \\
\hline$\geq 60$ & $15(18.5)$ & $10(66.7)$ & $5(33.3)$ & & $7(46.7)$ & $8(53.3)$ & \\
\hline$<60$ & $66(81.5)$ & $48(72.7)$ & $18(27.3)$ & & $23(34.8)$ & $43(65.2)$ & \\
\hline T status, n (\%) & & & & 0.242 & & & 0.458 \\
\hline $\mathrm{T} 1, \mathrm{~T} 2$ & $47(58.0)$ & $36(76.6)$ & $11(23.4)$ & & $19(40.4)$ & $28(59.6)$ & \\
\hline $\mathrm{T} 3, \mathrm{~T} 4$ & $34(42.0)$ & $22(64.7)$ & $12(35.3)$ & & $11(32.4)$ & $23(67.6)$ & \\
\hline $\mathrm{N}$ status, $\mathrm{n}(\%)$ & & & & $0.032^{\mathrm{a}}$ & & & $0.027^{\mathrm{a}}$ \\
\hline N0, N1 & $41(50.6)$ & $25(61.0)$ & $16(39.0)$ & & $20(48.8)$ & $21(51.2)$ & \\
\hline $\mathrm{N} 2, \mathrm{~N} 3$ & $40(49.4)$ & $33(82.5)$ & $7(17.5)$ & & $10(25.0)$ & $30(75.0)$ & \\
\hline AJCC stage, n (\%) & & & & 0.518 & & & $0.019^{\mathrm{a}}$ \\
\hline I, II & $16(19.8)$ & $13(81.3)$ & $3(18.7)$ & & $10(62.5)$ & $6(37.5)$ & \\
\hline III, IV & $65(80.2)$ & $45(69.2)$ & $20(30.8)$ & & $20(30.8)$ & $45(69.2)$ & \\
\hline
\end{tabular}

${ }^{\mathrm{a}} \mathrm{P}<0.05$. ROCK1, rho-associated coiled-coil-containing protein kinase 1; PIK3CA, phosphatidylinositol-4,5-bisphosphate 3-kinase catalytic subunit $\alpha$; low ROCK1/PIK3CA immunoexpression, $0-25 \%$ of cells stained; high ROCK1/PIK3CA immunoexpression, 26-100\% of cells stained.
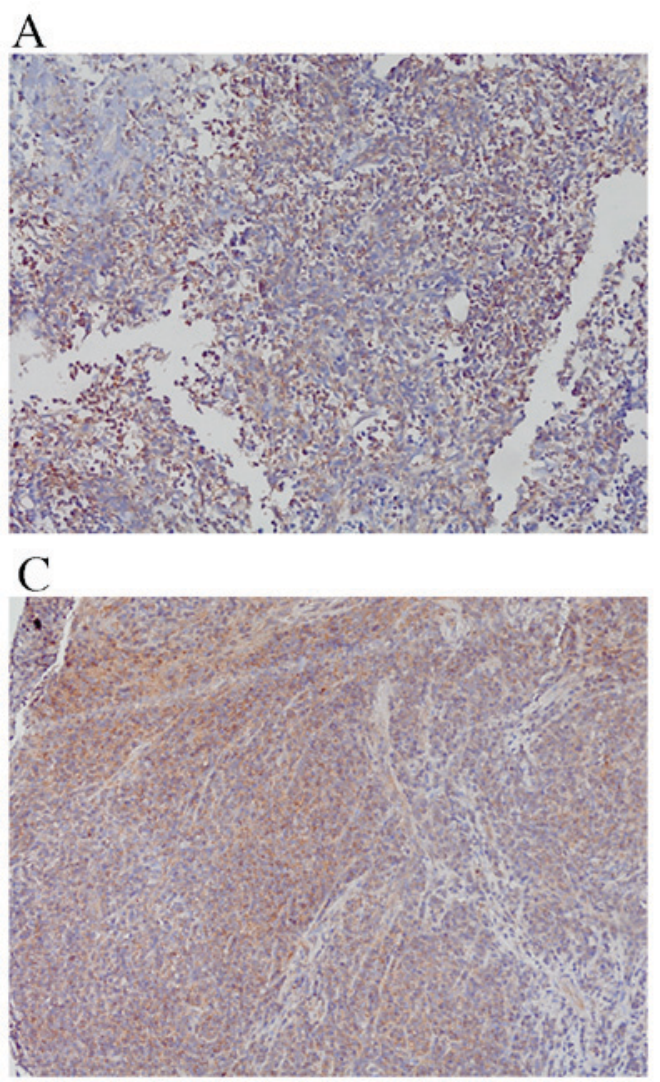

$\mathrm{B}$

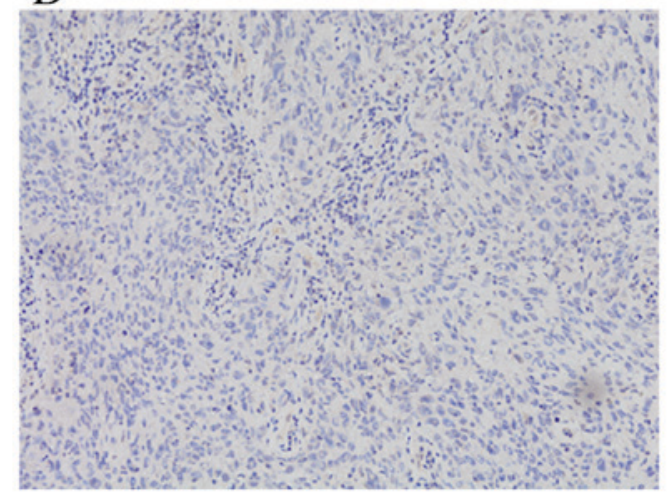

$\mathrm{D}$

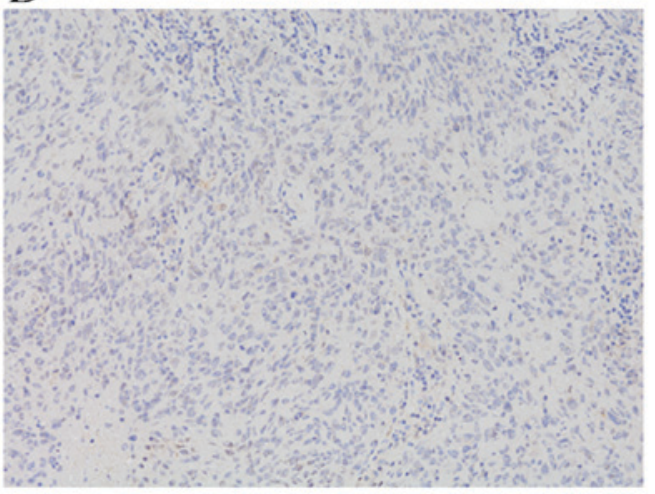

Figure 1. Representative images of immunohistochemical staining for ROCK1 and PIK3CA in tissue microarray sections prepared from nasopharyngeal carcinoma specimens (magnification, x400). (A) High expression of ROCK1 was observed in the cytoplasm. (B) Low expression of ROCK1 was observed in the cytoplasm. (C) High expression of PIK3CA was observed in the cytoplasm. (D) Low expression of PIK3CA was observed in the cytoplasm. ROCK1, rho-associated coiled-coil-containing protein kinase 1; PIK3CA, phosphatidylinositol-4,5-bisphosphate 3-kinase catalytic subunit $\alpha$. 
Table II. Correlation between the expression of ROCK1 and PIK3CA in 81 cases of nasopharyngeal carcinoma.

PIK3CA

\begin{tabular}{lcccr}
\cline { 3 - 4 } ROCK1 & Number of cases & Low, n (\% of ROCK1) & High, n (\% of ROCK1) & P-value \\
\hline Low & 58 & $27(46.6)$ & $31(53.4)$ & 0.01 \\
High & 23 & $3(13.0)$ & $20(87.0)$ & \\
\hline
\end{tabular}

ROCK1, rho-associated coiled-coil-containing protein kinase 1; PIK3CA, phosphatidylinositol-4,5-bisphosphate 3-kinase catalytic subunit $\alpha$; low ROCK1/PIK3CA immunoexpression, 0-25\% of cells stained; high ROCK1/PIK3CA immunoexpression, 26-100\% of cells stained.

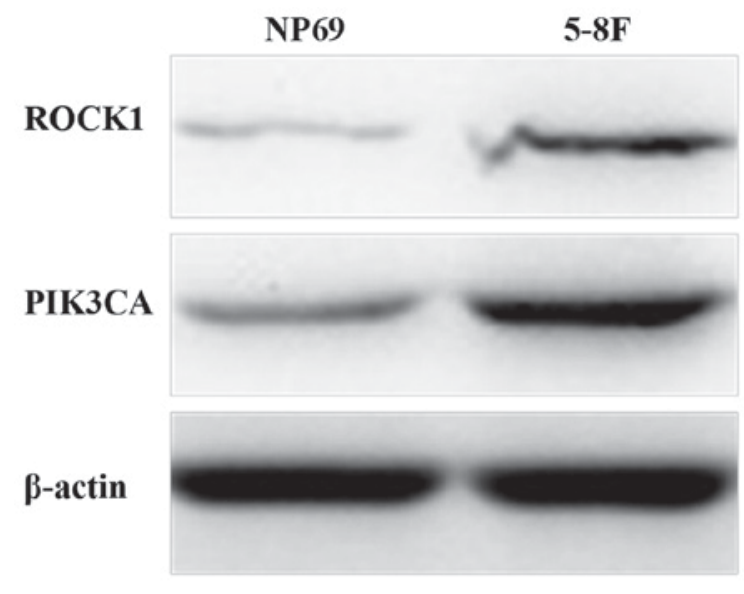

Figure 2. Western blot detection of ROCK1 and PI3KCA protein levels from NP69 and 5-8F cells. ROCK1, rho-associated coiled-coil-containing protein kinase 1; PIK3CA, phosphatidylinositol-4,5-bisphosphate 3-kinase catalytic subunit $\alpha$.

expression was observed in the cytoplasm, with high ROCK1 expression detected in $28.40 \%$ (23/81) of the NPC specimens and high PIK3CA expression detected in $62.96 \%$ (51/81) of the NPC specimens.

Expression of ROCK1 and PI3KCA in NPC cells. To assess the association between expressions of ROCK1 and PI3KCA and NPC, NP69 and 5-8F cells were used. ROCK1 and PIK3CA protein levels were demonstrated to be significantly higher in 5-8F cells compared with NP69 cells (Fig. 2). Expression of ROCK1 (relative to $\beta$-actin) in NP69 and 5-8F cells was $0.326 \pm 0.078$ and $1.191 \pm 0.114$, respectively $(\mathrm{P}<0.001)$. Expression of PIK3CA (relative to $\beta$-actin) in NP69 and 5-8F cells was $0.696 \pm 0.134$ and $1.159 \pm 0.144$, respectively $(P=0.015)$. Similar results were obtained from at least three independent western blots.

Association between ROCK1 and PIK3CA protein expression and the clinicopathological features of NPC. The associations between ROCK1 and PIK3CA expression and the clinicopathological features of NPC are displayed in Table I. High expression of ROCK1 was significantly associated with advanced $\mathrm{N}$ stage $(\mathrm{P}=0.032)$. High expression of PIK3CA was significantly associated with advanced $\mathrm{N}$ stage $(\mathrm{P}=0.027)$ and TNM cancer stage $(\mathrm{P}=0.019)$. Furthermore, $\chi^{2}$ and correlation analyses demonstrated that ROCK1 expression was significantly positively correlated with PIK3CA expression, $(\mathrm{P}=0.01$; $\mathrm{r}=0.313$; Table II). No statistically significant associations were observed between ROCK1 and PIK3CA expression and patient gender, age or T stage.

\section{Discussion}

NPC is a heterogeneous tumor type and patients with similar clinical and pathological features have varied outcomes, highlighting the underlying diversity of this disease (14). Therefore, it is necessary to identify effective prognostic factors that may represent potential molecular targets to develop effective therapeutic treatments for NPC.

Previous studies have suggested that high level expression of ROCK1 may promote cell invasion $(15,16)$. The ROCK pathway is one of the most important mechanisms of cellular invasion (17). When activated, Rho GTP binds to ROCK and the protein conformation of ROCK alters to fully expose the catalytic domain, allowing ROCK to phosphorylate downstream effector molecules (18). Increased expression of ROCK1 has frequently been observed in invasive and metastatic laryngeal squamous cell carcinoma, prostate cancer and several other malignant carcinomas, and it has been suggested that it serves a key role in promoting tumor invasion and metastasis $(19,20)$. In the present study, elevated expression of ROCK1 was observed in $28.40 \%$ of NPC specimens, and high ROCK1 expression was significantly associated with advanced $\mathrm{N}$ stage. $\mathrm{N}$ stage is the most important predictor of survival in NPC (21), reflecting the potential for the tumor to invade and metastasize. To the best of our knowledge, this is the first report detailing the association between ROCK1 and $\mathrm{N}$ category in NPC. The results of the present study suggest that ROCK1 may be associated with tumor metastases.

PIK3CA is associated with cancer growth, invasion and metastasis (22). PIK3CA mutations are typically detected in a wide range of cancers, including head and neck squamous cell carcinoma, colorectal cancer and breast cancer (23-27). In the present study, high levels of PIK3CA expression were detected in $62.96 \%$ of the NPC specimens. This indicates that PIK3CA may function as a tumor promoter in NPC. One of the aims of the present study was to assess the association between PIK3CA expression and the clinicopathological factors of NPC. Based on the results of the present study, it may be suggested that high PIK3CA expression is associated with advanced $\mathrm{N}$ stage and TNM stage.

Ehrenschwender et al (28) demonstrated that mutations in PIK3CA enabled tumor necrosis factor-related 
apoptosis-inducing ligand and Fas ligand to induce non-apoptotic, caspase-8-mediated ROCK1 activation. The present study demonstrated that high PIK3CA expression was significantly correlated with high ROCK1 expression. The results of the present study, together with those of Ehrenschwender et al (28), indicate that high expression of PIK3CA may promote tumor proliferation, malignant transformation and invasion via the ROCK pathway. Additionally, co-expression of these markers may have a more significant prognostic value than expression of one protein alone. A longer follow-up time is required to determine the overall survival of patients in the present study. Future research may include survival analysis between ROCK1 and PIK3CA expression and the overall survival of patients with NPC.

In conclusion, the results of the present study indicate that elevated expressions of ROCK1 and PIK3CA are correlated with the progression of NPC. The present study may provide a basis for future research into cancer therapy by employing these proteins as potential molecular targets. Further studies are required to elucidate the mechanisms by which ROCK1 and PIK3CA contribute to NPC.

\section{Acknowledgements}

The present study was supported by the National Natural Science Foundation of China (grant nos. 81274141, 81450051, 81573656 and 81403232), the Plans of Colleges and Universities in Jiangsu Province to Postgraduate Research and Innovation (grant no. KYZZ15-0368), the Foundation of SuBei People's Hospital (grant no. yzucms201409), and the Natural Science Foundation of Jiangsu Province (grant no. SBK2014021480).

\section{References}

1. Chang ET and Adami HO: The enigmatic epidemiology of nasopharyngeal carcinoma. Cancer Epidemiol Biomarkers Prev 15: 1765-1777, 2006.

2. Perri F, Dell'Oca I, Muto P, Schiavone C, Aversa C, Fulciniti F, Solla R, Scarpati GD, Buonerba C, Di Lorenzo G and Caponigro F: Optimal management of a patient with recurrent nasopharyngeal carcinoma. World J Clin Cases 2: 297-300, 2014

3. Wang T, Riaz N, Cheng S, Lu J and Lee N: Intensity-modulated radiation therapy for nasopharyngeal carcinoma: A review. J Radiat Oncol 1: 129-146, 2012.

4. Jin Y, Cai XY, Cai YC, Cao Y, Xia Q, Tan YT, Jiang WQ and Shi YX: To build a prognostic score model containing indispensible tumor markers for metastatic nasopharyngeal carcinoma in an epidemic area. Eur J Cancer 48: 882-888, 2012.

5. Lee AW, Ng WT, Chan YH, Sze H, Chan C and Lam TH: The battle against nasopharyngeal cancer. Radiother Oncol 104: 272-278, 2012

6. Zhang W, Zeng Z, Wei F, Chen P, Schmitt DC, Fan S, Guo X, Liang F, Shi L, Liu Z, et al: SPLUNC1 is associated with nasopharyngeal carcinoma prognosis and plays an important role in all-trans-retinoic acid-induced growth inhibition and differentiation in nasopharyngeal cancer cells. FEBS J 281: 4815-4829, 2014.

7. Li M, Li C, Li D, Xie Y, Shi J, Li G, Guan Y, Li M, Zhang P, Peng F, et al: Periostin, a stroma-associated protein, correlates with tumor invasiveness and progression in nasopharyngeal carcinoma. Clin Exp Metastasis 29: 865-877, 2012.

8. Newell-Litwa KA, Badoual M, Asmussen H, Patel H, Whitmore L and Horwitz AR: ROCK1 and 2 differentially regulate actomyosin organization to drive cell and synaptic polarity. J Cell Biol 210: 225-242, 2015.

9. Shi Y, Pontrello CG, DeFea KA, Reichardt LF and Ethell IM: Focal adhesion kinase acts downstream of EphB receptors to maintain mature dendritic spines by regulating cofilin activity. J Neurosci 29: 8129-8142, 2009.
10. Lai K, Killingsworth $\mathrm{MC}$ and Lee CS: Gene of the month: PIK3CA. J Clin Pathol 68: 253-257, 2015.

11. Zhao Q, Zhang B, Shao Y, Chen L, Wang X, Zhang Z, Shu Y and Guo R: Correlation between the expression levels of miR-1 and PIK3CA in non-small-cell lung cancer and their relationship with clinical characteristics and prognosis. Future Oncol 10: 49-57, 2014.

12. Edge SB, Byrd DR, Compton CC, Fritz AG, Greene F and Trotti A: Pharynx. In: AJCC Cancer Staging Manual. 7th Edition. Springer Science \& Business Media LLC, NY, pp41-49, 2010.

13. Bradford MM: A rapid and sensitive method for the quantitation of microgram quantities of protein utilizing the principle of protein-dye binding. Anal Biochem 72: 248-254, 1976.

14. Zeng Z, Huang H, Zhang W, Xiang B, Zhou M, Zhou Y, Ma J, Yi M, Li X, Li X, et al: Nasopharyngeal carcinoma: Advances in genomics and molecular genetics. Sci China Life Sci 54: 966-975, 2011.

15. Vigil D, Kim TY, Plachco A, Garton AJ, Castaldo L, Pachter JA, Dong H, Chen X, Tokar B, Campbell SL and Der CJ: ROCK1 and ROCK2 are required for non-small cell lung cancer anchorage-independent growth and invasion. Cancer Res 72: 5338-5347, 2012.

16. Chen J, Ye L, Zhang L and Jiang WG: Placenta growth factor, PLGF, influences the motility of lung cancer cells, the role of Rho associated kinase, Rock1. J Cell Biochem 105: 313-320, 2008.

17. Tripathi V, Popescu NC and Zimonjic DB: DLC1 induces expression of E-cadherin in prostate cancer cells through Rho pathway and suppresses invasion. Oncogene 33: 724-733, 2014.

18. Croft DR, Crighton D, Samuel MS, Lourenco FC, Munro J, Wood J, Bensaad K, Vousden KH, Sansom OJ, Ryan KM and Olson MF: p53-mediated transcriptional regulation and activation of the actin cytoskeleton regulatory RhoC to LIMK2 signaling pathway promotes cell survival. Cell Res 21: 666-682, 2011.

19. Zhang J, He X, Ma Y, Liu Y, Shi H, Guo W and Liu L: Overexpression of ROCK1 and ROCK2 inhibits human laryngeal squamous cell carcinoma. Int J Clin Exp Pathol 8: 244-251, 2015.

20. Zhang C, Zhang S, Zhang Z, He J, Xu Y and Liu S: ROCK has a crucial role in regulating prostate tumor growth through interaction with c-Myc. Oncogene 33: 5582-5591, 2014.

21. Chen KW, Wang WY, Liang WM, Twu CW, Chao JY, Liang KL, Wu CT, Jiang RS, Shih YT and Lin JC: The volume of retropharyngeal nodes predicts distant metastasis in patients with advanced nasopharyngeal carcinoma. Oral Oncol 47: 1171-1175, 2011.

22. Vivanco I and Sawyers CL: The phosphatidylinositol 3-Kinase AKT pathway in human cancer. Nat Rev Cancer 2: 489-501, 2002.

23. Psyrri A, Seiwert TY and Jimeno A: Molecular pathways in head and neck cancer: EGFR, PI3K and more. Am Soc Clin Oncol Educ Book: 246-255, 2013.

24. Chang YS, Hsu HT, Ko YC, Yeh KT, Chang SJ, Lin CY and Chang JG: Combined mutational analysis of RAS, BRAF, PIK3CA, and TP53 genes in Taiwanese patients with oral squamous cell carcinoma. Oral Surg Oral Med Oral Pathol Oral Radiol 118: 110-116.e1, 2014.

25. Qiu W, Tong GX, Manolidis S, Close LG, Assaad AM and Su GH: Novel mutant-enriched sequencing identified high frequency of PIK3CA mutations in pharyngeal cancer. Int J Cancer 122: 1189-1194, 2008.

26. Barault L, Veyrie N, Jooste V, Lecorre D, Chapusot C, Ferraz JM, Lièvre A, Cortet M, Bouvier AM, Rat $\mathrm{P}$, et al: Mutations in the RAS-MAPK, PI(3)K (phosphatidylinositol-3-OH kinase) signaling network correlate with poor survival in a population-based series of colon cancers. Int J Cancer 122: 2255-2259, 2008.

27. Kalinsky K, Jacks LM, Heguy A,Patil S, Drobnjak M, Bhanot UK, Hedvat CV, Traina TA, Solit D, Gerald W and Moynahan ME: PIK3CA mutation associates with improved outcome in breast cancer. Clin Cancer Res 15: 5049-5059, 2009.

28. Ehrenschwender M, Siegmund D, Wicovsky A, Kracht M, Dittrich-Breiholz O, Spindler V, Waschke J, Kalthoff H, Trauzold A and Wajant H: Mutant PIK3CA licenses TRAIL and CD95L to induce non-apoptotic caspase-8-mediated ROCK activation. Cell Death Differ 17: 1435-1447, 2010. 